\title{
C-Cyanation with 1-Cyanobenzotriazole
}

\author{
Alan R. Katritzky*, Rufine Akue-Gedu, and Anatoliy V. Vakulenko \\ Center for Heterocyclic Compounds, Department of Chemistry, University of Florida, \\ Gainesville, FL 32611-7200 \\ E-mail: Katritzky@chem.ufl.edu
}

\section{Dedicated to Professor Ernst Anders on the occasion of his $65^{\text {th }}$ birthday}

\begin{abstract}
1-Cyanobenzotriazole is a convenient source of $\mathrm{NC}^{+}$for $\mathrm{C}$-cyanation with in situ-generated $\mathrm{sp}^{3}$ and $\mathrm{sp}^{2}$ carbanions providing $\alpha$-cyano-sulfones, -ketones, -alkanecarboxylate esters, -cyanides, alkylheterocycles, -diarylmethanes and heterylcarbonitriles in good to high yields (55-78\%).
\end{abstract}

Keywords: Nitriles, 1-cyanobenzotriazole, $C$-cyanation, electrophilic substitution reaction

\section{Introduction}

Most reactions to introduce the cyano group employ the cyanide anion $\mathrm{NC}^{-}$. Electrophilic reaction with $\mathrm{NC}^{+}$donors are more rare: most are electrophilic substitution reactions of organometallic reagents ${ }^{1}$ with tosyl cyanide, ${ }^{2 a, b}$ 2-chlorobenzyl thiocyanate, ${ }^{3}$ thiocyanogen, ${ }^{4} 2$ cyanopyridazin-3(2H)-ones, ${ }^{5}$ cyanogen halides, ${ }^{6}$ and 1-cyanoimidazole. ${ }^{7}$ These show problems with stability, lack of reactivity, poor solubility, corrosiveness, availability, toxicity, cost, and/or complicated preparative procedures.

1-Cyanobenzotriazole 1 has been used previously in our group for cyanation of amines and thiols. ${ }^{8}$ More recently, Cava's group reported the C-cyanating ability of $\mathbf{1}$ for arylacetonitriles ${ }^{9}$ and aromatic and heteroaromatic compounds, ${ }^{1}$ where moderate to good yields were obtained for cyanation of simple alkyl- and arenyl- carbanions and the thiophene ring system. In the present work, we have expanded the application of 1-cyanobenzotriazole to the preparation of $\alpha$-cyanosulfones, -ketones, -alkanecarboxylate esters, -cyanides, -alkylheterocycles, -diarylmethanes and heterylcarbonitriles. 


\section{Results and Discussion}

1-Cyanobenzotriazole 1 was readily prepared in high yield (92\%) following a slightly modified literature procedure ${ }^{8}$ by the treatment of benzotriazole with sodium hydride followed by addition of cyanogen bromide (Scheme 1). Use of the sodium salt of benzotriazole, prepared in situ, affords the pure 1-cyanobenzotriazole $\mathbf{1}$ after simple filtration and evaporation of solvent. Alternative procedures require purification by sublimation. ${ }^{8,9}$
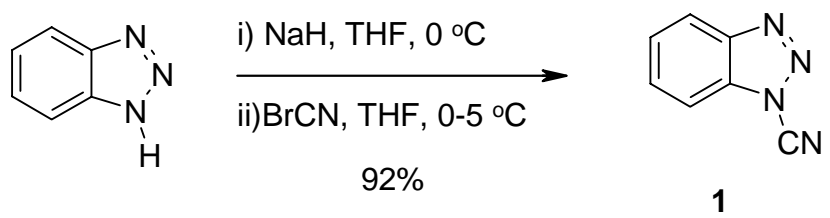

\section{Scheme 1}

Synthesis of nitriles derivatives. The general utility of 1-cyanobenzotriazole for incorporation of the nitrile functional group into a diverse series of functionalized compounds 2 was investigated by reaction of the corresponding carbanions, again generated in situ, with cyanating reagent 1. For optimum yields, reactions of lithium diisopropylamide (LDA, prepared as detailed in method A) with substrates $\mathbf{2 a}-\mathbf{j}$ required room temperature for $2 \mathrm{~h}$ followed by the addition of 1-cyanobenzotriazole at $0{ }^{\circ} \mathrm{C}$. Then the reaction mixture was allowed to react at room temperature overnight and was quenched with a saturated solution of ammonium chloride. Extraction with ethyl acetate and purification by silica gel column chromatography provided $\alpha$ cyanoalkyl derivatives in average $65 \%$ yield (Scheme 2, Table 1). Nitriles 3k,l were prepared by method $\mathrm{B}$, which requires 2.2 molar equiv of $n-\mathrm{BuLi}$ and $12 \mathrm{~h}$ at room temperature for generation of the corresponding lithio derivatives. Treatment with 1-cyanobenzotriazole 1 gave the desired products $3 \mathbf{k}, \mathbf{l}$ in 65 and $70 \%$ yields.

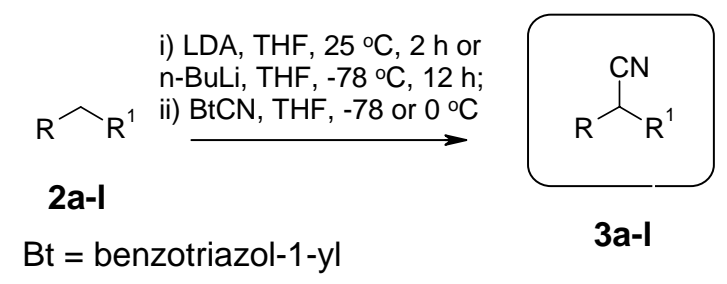

${ }^{\mathrm{a}}$ For designation of $\mathrm{R}$ and $\mathrm{R}^{1}$ in $\mathbf{2}$ and $\mathbf{3}$ see Table 1.

\section{Scheme 2}

Lithio derivatives (generated in situ by treating the corresponding heterocycles $2 \mathbf{m}, \mathbf{n}$ with 1 molar equiv of $n$-BuLi for $1 \mathrm{~h}$ (for $3 \mathrm{~m}$ ) or for $12 \mathrm{~h}$ (for $3 \mathbf{n}$ )) were treated with 1- 
cyanobenzotriazole 1 in dry THF at $-78{ }^{\circ} \mathrm{C}$. The reaction mixture was allowed to warm to room temperature while stirring overnight and afforded, after workup, heterylcarbonitriles $\mathbf{3 m , n}$ in 55$65 \%$ isolated yields (Scheme 3 and Table 1).

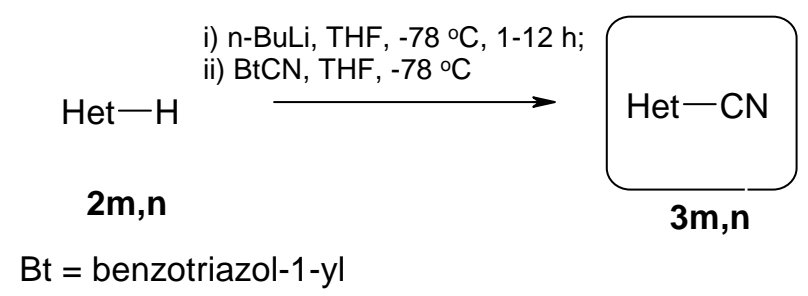

${ }^{\text {a }}$ For designation of Het in $\mathbf{2}$ and $\mathbf{3}$ see Table 1.

\section{Scheme 3}

Table 1. Preparation nitriles 3a-n

\begin{tabular}{ccccc}
\hline Product & $\mathrm{R}$ or Het of $\mathbf{2}$ and $\mathbf{3}$ & $\mathrm{R}^{1}$ of $\mathbf{2}$ and $\mathbf{3}$ & Yield (\%) of $\mathbf{3}$ & Method \\
\hline 3a & $\mathrm{Ph}$ & $\mathrm{CN}$ & 70 & $\mathrm{~A}$ \\
3b & $\mathrm{Bz}$ & $\mathrm{CN}$ & 65 & $\mathrm{~A}$ \\
3c & $\mathrm{Ph}$ & $\mathrm{CO}_{2} \mathrm{Et}$ & 70 & $\mathrm{~A}$ \\
3d & $\mathrm{Bz}$ & $\mathrm{CO}_{2} \mathrm{Et}$ & 67 & $\mathrm{~A}$ \\
3e & 1-naphthyl & $\mathrm{CO}_{2} \mathrm{Me}$ & 65 & $\mathrm{~A}$ \\
3f & 2-tolyl & $\mathrm{SO}_{2} \mathrm{Ph}$ & 75 & $\mathrm{~A}$ \\
3g & $\mathrm{Ph}$ & $\mathrm{SO}_{2} \mathrm{Ph}$ & 78 & $\mathrm{~A}$ \\
3h & $\mathrm{Bz}$ & $\mathrm{SO}_{2} \mathrm{Ph}$ & 65 & $\mathrm{~A}$ \\
3i & 4-anisyl & $\mathrm{COMe}_{3}$ & 35 & $\mathrm{~A}$ \\
3j & $\mathrm{Ph}$ & $\mathrm{COPh}_{3}$ & 62 & $\mathrm{~A}$ \\
3k & $\mathrm{Ph}$ & $\mathrm{Ph}$ & 65 & $\mathrm{~B}$ \\
3l & 2-pyridyl & $\mathrm{Me}$ & 70 & $\mathrm{~B}$ \\
3m & 2-ethyl-5-furyl & - & 65 & $\mathrm{~B}$ \\
3n & 1-methyl-2-pyrrolyl & - & 55 & $\mathrm{~B}$ \\
\hline
\end{tabular}

The cyanation of ketone $2 \mathbf{i}$ under the standard lithiation conditions (method A) provided a mixture of two isomers $3 \mathbf{i}$ and $\mathbf{4}$ due to the generation of the two corresponding anions on treatment of methyl 2-(4-methoxyphenyl)acetate $2 \mathbf{i}$ with LDA $^{10}$ (Scheme 4). Our attempts to improve the yield of methyl 2-cyano-2-(4-methoxyphenyl)acetate 3i using shorter reaction times, lower temperatures and $n$-BuLi were unsuccessful. A variable amount of cyanomethyl 2-(4methoxyphenyl)acetate 4 beside the major product 3i was always detected in the reaction mixture. 


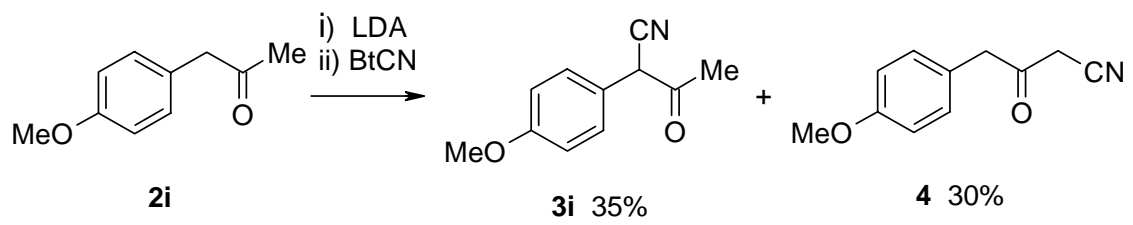

\section{Scheme 4}

In summary, the utility of 1-cyanobenzotriazole for preparation of a broad variety of heterocyclic and functionalized $\alpha$-nitriles has been established as a general and efficient methodology. 1-Cyanobenzotriazole is an advantageous cyanation reagent because it is a stable, non-volatile, crystalline solid that limits the risk of possible toxic exposure.

\section{Experimental Section}

General Procedures. Melting points were determined using a Bristoline hot-stage microscope and are uncorrected. ${ }^{1} \mathrm{H}(300 \mathrm{MHz})$ and ${ }^{13} \mathrm{C}(75 \mathrm{MHz})$ NMR spectra were recorded on a 300 $\mathrm{MHz}$ NMR spectrometer in chloroform- $d$ solution. Elemental and mass spectroscopy analyses were performed by the Analytical Laboratories, Dept. of Chem., University of Florida. THF was distilled from sodium-benzophenone ketal prior to use. All the reactions were performed under a nitrogen atmosphere. Column chromatography was conducted with silica gel (200-425 mesh).

1-Cyano-1H-1,2,3-benzotriazole (1). Benzotriazole (10 g, $84 \mathrm{mmol})$ was dissolved in anhydrous THF $(150 \mathrm{ml})$. The solution was cooled to $0{ }^{\circ} \mathrm{C}$ and $60 \%$ sodium hydride $(3.36 \mathrm{~g}, 92.4$ $\mathrm{mmol}$ ) was added. The mixture was allowed to react at the same temperature for 30 minutes. A solution of cyanogen bromide $(9.7 \mathrm{~g}, 92.4 \mathrm{mmol})$ in dry THF $(25 \mathrm{~mL})$ was added rapidly to a vigorously stirred solution of sodium benzotriazole. The reaction mixture was allowed to react at ambient temperature for $1.5 \mathrm{~h}$. The precipitate was filtered off and washed with THF. The filtrate was evaporated and the residue was dissolved in ethyl acetate and washed with water. The organic phase was dried over magnesium sulfate, filtered and the solvent was removed under reduced pressure to afford 1-cyanobenzotriazole as a colorless powder $(92 \%), \mathrm{mp} 74-76{ }^{\circ} \mathrm{C}$ (lit. ${ }^{8}$ mp 73-76 $\left.{ }^{\circ} \mathrm{C}\right) ;{ }^{1} \mathrm{H}$ NMR $\delta 8.23(\mathrm{~d}, J=8.4 \mathrm{~Hz}, 1 \mathrm{H}), 7.76-7.87(\mathrm{~m}, 2 \mathrm{H}), 7.58-7.68(\mathrm{~m}, 1 \mathrm{H}) ;{ }^{13} \mathrm{C}$ NMR $\delta$ 143.3, 132.7, 131.6, 126.8, 121.4, 109.5, 103.7.

\section{General procedure of preparation of sulfones $2 f-h$}

To ethanol $(100 \mathrm{~mL})$ was added phenyl sulfonyl sodium salt $(60.9 \mathrm{mmol})$ and the respective benzylbromide $(60.9 \mathrm{mmol})$. The mixture was refluxed for 3 hours. The solvent was evaporated and the solid was washed with water and with isopropyl ether to afford compounds $\mathbf{3 f}-\mathbf{h}$. 
2-Methylbenzyl phenyl sulfone (2f). White prisms (75\%), mp 73-74 ${ }^{\circ} \mathrm{C}$ (lit. $\left.{ }^{11} \mathrm{mp} 73-75{ }^{\circ} \mathrm{C}\right)$; ${ }^{1} \mathrm{H}$ NMR $\delta$ 7.58-7.66 (m, 3H), 7.43-7.48 (m, 2H), 7.12-7.14 (m, 2H), 6.83-6.90 (m, 2H), 4.27 (s, 2H), 2.26 (s, 3H); ${ }^{13} \mathrm{C}$ NMR $\delta 138.2,137.8,133.6,131.5,129.4,128.7,128.5,128.3,127.73$, 127.72, 62.8, 21.1. Anal. Calcd for $\mathrm{C}_{14} \mathrm{H}_{14} \mathrm{O}_{2} \mathrm{~S}$ : C, 68.26; H, 5.73; Found: C, 68.14; H, 5.75.

Benzyl phenyl sulfone (2g). Colorless prisms (70\%), mp 148-150 ${ }^{\circ} \mathrm{C}$ (lit. ${ }^{12} \mathrm{mp} 146-147{ }^{\circ} \mathrm{C}$ ); ${ }^{1} \mathrm{H}$ NMR $\delta$ 7.63-7.76 (m, 3H), $7.43(\mathrm{~m}, 2 \mathrm{H}), 7.33-7.22(\mathrm{~m}, 3 \mathrm{H}), 7.07(\mathrm{~m}, 2 \mathrm{H}), 4.31(\mathrm{~s}, 2 \mathrm{H}) ;{ }^{13} \mathrm{C}$ NMR $\delta 137.7,133.6,130.7,128.8,128.7,128.5,128.4,128.0$, 62.7. Anal. Calcd for $\mathrm{C}_{13} \mathrm{H}_{12} \mathrm{O}_{2} \mathrm{~S}$ : C, 67.22; H, 5.21; Found: C, 66.85; H, 5.21.

Phenethyl phenyl sulfone (2h). White prisms (65\%), mp 53-54 ${ }^{\circ} \mathrm{C}$ (lit. $\left.{ }^{12} \mathrm{mp} 53-56{ }^{\circ} \mathrm{C}\right) ;{ }^{1} \mathrm{H}$ NMR $\delta$ 7.96-7.93 (m, 2H), 7.67 (m, 1H), 7.58 (m, 2H), 7.30-7.18 (m, 3H), 7.11 (m, 2H), 3.40$3.33(\mathrm{~m}, 2 \mathrm{H}), 3.10-3.01(\mathrm{~m}, 2 \mathrm{H}) ;{ }^{13} \mathrm{C}$ NMR $\delta 138.9,137.4,133.8,129.3,128.8,128.2,128.1$, 126.9, 57.5, 28.7. Anal. Calcd for $\mathrm{C}_{14} \mathrm{H}_{14} \mathrm{O}_{2} \mathrm{~S}$ : C, 68.26; H, 5.73; Found: C, 67.94; H, 5.76.

\section{General procedures for $C$-cyanation}

Method A. A solution of diisopropylamine (12.0 mmol, $1.68 \mathrm{~mL})$ in THF $(15.0 \mathrm{~mL})$ was treated with $n$-butyllithium $(2.5 \mathrm{M}$ in $n$-hexane, $12.0 \mathrm{mmol}, 4.8 \mathrm{~mL})$, at $0{ }^{\circ} \mathrm{C}$, under a nitrogen atmosphere. After $30 \mathrm{~min}$ at $0{ }^{\circ} \mathrm{C}$, a solution of $2(6 \mathrm{mmol})$ in THF $(15 \mathrm{~mL})$ was added dropwise. The mixture was stirred at room temperature for $2 \mathrm{~h}$, cooled to $0{ }^{\circ} \mathrm{C}$ and then a solution of 1cyanobenzotriazole $(0.91 \mathrm{~g}, 6.3 \mathrm{mmol})$ in THF $(10 \mathrm{~mL})$ was slowly added. The mixture was allowed to warm to room temperature while stirring overnight and then was poured into a saturated solution of ammonium chloride $(60 \mathrm{~mL})$. The product was extracted with ethyl acetate $(30 \mathrm{~mL} \times 3)$. The combined organic extracts were washed with brine and dried over $\mathrm{MgSO}_{4}$. After evaporation under vacuum, the residue was purified by column chromatography $\left(\mathrm{CH}_{2} \mathrm{Cl}_{2}\right.$ or heptane/ethyl acetate: 10/1) to afford the desired functionalized $\alpha$-nitriles 3a-j and $\mathbf{4}$.

2-Phenylmalononitrile (3a). Colorless prisms (70\%), mp 65-67 ${ }^{\circ} \mathrm{C}$, (lit. ${ }^{3} \mathrm{mp} 67-68{ }^{\circ} \mathrm{C}$ ); ${ }^{1} \mathrm{H}$ NMR $\delta 7.50(\mathrm{~s}, 5 \mathrm{H}), 5.09(\mathrm{~s}, 1 \mathrm{H}) ;{ }^{13} \mathrm{C}$ NMR $\delta$ 130.4, 130.1, 127.2, 126.2, 111.9, 28.1. Anal. Calcd for $\mathrm{C}_{9} \mathrm{H}_{6} \mathrm{~N}_{2}$ : C, 76.04; H, 4.25; N, 19.71. Found: C, 75.99; H, 4.05; N, 19.59.

2-Benzylmalononitrile (3b). Colorless prisms (65\%), mp 86-88 ${ }^{\circ} \mathrm{C}$, (lit. $\left.{ }^{13} \mathrm{mp} 80-81{ }^{\circ} \mathrm{C}\right) ;{ }^{1} \mathrm{H}$ NMR $\delta$ 7.45-7.40 (m, 3H), 7.36-7.30 (m, 2H), $3.91(\mathrm{t}, J=7.0 \mathrm{~Hz}, 1 \mathrm{H}), 3.29(\mathrm{~d}, J=7.0 \mathrm{~Hz}, 2 \mathrm{H})$; ${ }^{13} \mathrm{C}$ NMR $\delta 132.9,129.3,129.1,128.8,112.1,36.7,25.0$. Anal. Calcd for $\mathrm{C}_{10} \mathrm{H}_{8} \mathrm{~N}_{2}: \mathrm{C}, 76.90 ; \mathrm{H}$, 5.16; N, 17.94. Found: C, 76.65; H, 5.12; N, 18.04 .

Ethyl 2-cyano-2-phenylacetate (3c). ${ }^{14}$ Yellow oil (70\%); ${ }^{1} \mathrm{H}$ NMR $\delta$ 7.45-7.46 (m, 5H), 4.72 (s, $1 \mathrm{H}), 4.23$ (q, $J=7.2 \mathrm{~Hz}, 2 \mathrm{H}), 1.27$ (t, $J=7.2 \mathrm{~Hz}, 3 \mathrm{H}) ;{ }^{13} \mathrm{C}$ NMR $\delta 164.9,129.9,129.2,129.1$, 127.8, 115.6, 63.2, 43.6, 13.8. Anal. Calcd for $\mathrm{C}_{11} \mathrm{H}_{11} \mathrm{NO}_{2}$ : C, 69.82; H, 5.86; N, 7.40. Found: C, $70.14 ; \mathrm{H}, 6.18 ; \mathrm{N}, 7.13$.

Ethyl 2-cyano-3-phenylpropanoate (3d). ${ }^{13}$ Yellow oil $(67 \%) ;{ }^{1} \mathrm{H}$ NMR $\delta 7.37-7.28(\mathrm{~m}, 5 \mathrm{H})$, 4.24 (q, $J=7.1 \mathrm{~Hz}, 2 \mathrm{H}), 3.72$ (dd, $J=8.5,5.9 \mathrm{~Hz}, 1 \mathrm{H}), 3.20$ (dd, $J=13.9,8.5 \mathrm{~Hz}, 1 \mathrm{H}), 3.27$ (dd, $J=13.9,8.5 \mathrm{~Hz}, 1 \mathrm{H}), 1.27(\mathrm{t}, J=7.1 \mathrm{~Hz}, 3 \mathrm{H}) ;{ }^{13} \mathrm{C}$ NMR $\delta 165.5,135.2,129.1,129.0,128.8$, 127.8, 116.1, 62.9, 39.7, 35.7, 13.9. Anal. Calcd for $\mathrm{C}_{12} \mathrm{H}_{13} \mathrm{NO}_{2}$ : C, 70.92; H, 6.45; N, 6.89. Found: C, 71.24; H, 6.83; N, 7.24. 
Methyl 2-cyano-2-(1-naphthyl)acetate (3e). Colorless prisms $(65 \%)$, mp 90-92 ${ }^{\circ} \mathrm{C}$ (lit. ${ }^{15} \mathrm{mp}$ 89-90 $\left.{ }^{\circ} \mathrm{C}\right) ;{ }^{1} \mathrm{H}$ NMR $\delta 8.00(\mathrm{~d}, J=8.2 \mathrm{~Hz}, 1 \mathrm{H}), 7.92(\mathrm{~d}, J=8.2 \mathrm{~Hz}, 2 \mathrm{H}), 7.71(\mathrm{~d}, J=7.1 \mathrm{~Hz}$, $1 \mathrm{H}), 7.64-7.49(\mathrm{~m}, 3 \mathrm{H}), 5.39(\mathrm{~s}, 1 \mathrm{H}), 3.78(\mathrm{~s}, 3 \mathrm{H}) ;{ }^{13} \mathrm{C} \mathrm{NMR} \delta 165.5,133.9,130.3,130.2,129.1$, 127.6, 127.5, 126.4, 126.0, 125.4, 122.4, 115.7, 53.9, 41.2. Anal. Calcd for $\mathrm{C}_{14} \mathrm{H}_{11} \mathrm{NO}_{2}$ : C, 74.65; H, 4.92; N, 6.22. Found: C, 74.40; H, 4.92; N, 6.19.

2-(2-Methylphenyl)-2-(phenylsulfonyl)acetonitrile (3f). Colorless prisms (75\%), mp 130-132 ${ }^{\circ} \mathrm{C}$ (lit. $\left.{ }^{16} \mathrm{mp} 133-134{ }^{\circ} \mathrm{C}\right) ; .{ }^{1} \mathrm{H}$ NMR $\delta$ 7.71-7.75 (m, 3H), 7.51-7.57 (m, 2H), 7.23-7.26 (m, 2H), 7.04-7.09 (m, 2H), 5.09 (s, 1H), 2.32 (s, 3H); ${ }^{13} \mathrm{C}$ NMR $\delta$ 139.0, 135.2, 134.3, 131.3, 130.3, 130.1, 129.1, 128.8, 126.8, 125.1, 113.5, 63.0, 21.2. Anal. Calcd for $\mathrm{C}_{15} \mathrm{H}_{13} \mathrm{NO}_{2} \mathrm{~S}$ : C, 66.40; $\mathrm{H}$, 4.83; N, 5.16. Found: C, 66.39; H, 4.73; N, 5.04.

2-Phenyl-2-(phenylsulfonyl)acetonitrile (3g). Colorless prisms $(70 \%)$, mp $151-152{ }^{\circ} \mathrm{C}$ (lit. $^{14}$ mp 147-148 $\left.{ }^{\circ} \mathrm{C}\right) ;{ }^{1} \mathrm{H}$ NMR $\delta$ 7.74-7.71 (m, 3H), $7.53(\mathrm{~m}, 2 \mathrm{H}), 7.44(\mathrm{~m}, 1 \mathrm{H}), 7.37$ (m, 2H), 7.27 (m, 2H), 5.14 (s, 1H); ${ }^{13} \mathrm{C}$ NMR $\delta 135.3,134.2,130.5,130.0,129.7,129.2,129.0,125.3,113.4$, 63.0. Anal. Calcd for $\mathrm{C}_{14} \mathrm{H}_{11} \mathrm{NO}_{2} \mathrm{~S}: \mathrm{C}, 65.35 ; \mathrm{H}, 4.31 ; 5.44$. Found: $\mathrm{C}, 65.42 ; \mathrm{H}, 4.30 ; \mathrm{N}, 5.43$.

3-Phenyl-2-(phenylsulfonyl)propanenitrile (3h). Yellow prisms $(65 \%), \mathrm{mp} 80-82{ }^{\circ} \mathrm{C}$ (lit. ${ }^{17} \mathrm{mp}$ 76-78 $\left.{ }^{\circ} \mathrm{C}\right) ;{ }^{1} \mathrm{H}$ NMR $\delta 8.10-8.05(\mathrm{~m}, 2 \mathrm{H}), 7.82-7.77(\mathrm{~m}, 1 \mathrm{H}), 7.70-7.65(\mathrm{~m}, 2 \mathrm{H}), 7.38-7.26(\mathrm{~m}$, $5 \mathrm{H}), 4.07$ (dd, $J=11.8,3.7 \mathrm{~Hz}, 1 \mathrm{H}), 3.60(\mathrm{dd}, J=13.6,3.7 \mathrm{~Hz}, 1 \mathrm{H}), 3.09$ (dd, $J=13.3,11.8 \mathrm{~Hz}$, $1 \mathrm{H}) ;{ }^{13} \mathrm{C}$ NMR $\delta 135.5,133.5,129.8,129.7,129.4,129.1,128.2,113.7,59.5,32.7$. Anal. Calcd for $\mathrm{C}_{15} \mathrm{H}_{13} \mathrm{NO}_{2} \mathrm{~S}: \mathrm{C}, 66.40 ; \mathrm{H}, 4.83 ; \mathrm{N}, 5.16$. Found: C, 66.45; H, 4.80; N, 5.10.

Methyl 2-cyano-2-(4-methoxyphenyl)acetate (3i). Yellow microcrystals (35\%), mp 80-82 ${ }^{\circ} \mathrm{C}$ (lit. $\left.{ }^{18} \mathrm{mp} 75-76{ }^{\circ} \mathrm{C}\right) ;{ }^{1} \mathrm{H}$ NMR $\delta 7.31(\mathrm{~d}, J=8.8 \mathrm{~Hz}, 2 \mathrm{H}), 6.95(\mathrm{~d}, J=8.8 \mathrm{~Hz}, 2 \mathrm{H}), 4.63(\mathrm{~s}, 1 \mathrm{H})$, 3.83 (s, 3H), 2.25 (s, 3H); ${ }^{13} \mathrm{C}$ NMR $\delta 196.8,160.3,129.2,121.5,116.4,115.1,55.4,50.8,26.8$. Anal. Calcd for $\mathrm{C}_{11} \mathrm{H}_{11} \mathrm{NO}_{2}$ : C, 69.83; H, 5.86; N, 7.40. Found: C, 69.83; H, 5.85; N, 7.32.

3-Oxo-2,3-diphenylpropanenitrile (3j). Colorless prisms $(62 \%), \mathrm{mp} 84-86{ }^{\circ} \mathrm{C}$ (lit. ${ }^{19} \mathrm{mp} 89-90$ $\left.{ }^{\circ} \mathrm{C}\right) ;{ }^{1} \mathrm{H}$ NMR $\delta 7.95(\mathrm{~d}, J=7.6 \mathrm{~Hz}, 2 \mathrm{H}), 7.63-7.58(\mathrm{~m}, 1 \mathrm{H}), 7.49-7.36(\mathrm{~m}, 7 \mathrm{H}), 5.60(\mathrm{~s}, 1 \mathrm{H}) ;{ }^{13} \mathrm{C}$ NMR $\delta 188.8,135.5,134.5,133.6,129.7,129.3,129.0,128.3,126.2,116.5,46.7$. Anal. Calcd for $\mathrm{C}_{15} \mathrm{H}_{11} \mathrm{NO}$ : C, 81.43; H, 5.01; N, 6.33. Found: C, 81.17; H, 5.15; N, 6.06.

Cyanomethyl 2-(4-methoxyphenyl)acetate (4). Yellow microcrystals (30\%), mp 82-84 ${ }^{\circ} \mathrm{C}$ (lit. ${ }^{20} \mathrm{mp} \mathrm{83.5-84.5}{ }^{\circ} \mathrm{C}$ ); ${ }^{1} \mathrm{H}$ NMR $\delta 7.14(\mathrm{~d}, J=8.7 \mathrm{~Hz}, 2 \mathrm{H}), 6.90$ (d, $\left.J=8.7 \mathrm{~Hz}, 2 \mathrm{H}\right), 3.81$ (s, 3H), 3.80 (s, 2H), 3.45 (s, 2H). ${ }^{13} \mathrm{C}$ NMR $\delta$ 195.6, 159.3, 130.5, 123.7, 114.7, 114.2, 55.3, 48.4, 30.9. Anal. Calcd for $\mathrm{C}_{11} \mathrm{H}_{11} \mathrm{NO}_{2}$ : C, 69.83; H, 5.86; N, 7.40. Found: $\mathrm{C}, 70.08 ; \mathrm{H}, 5.78 ; \mathrm{N}, 7.48$.

Method B. $n$-Butyl lithium (7 mmol (for $3 \mathbf{m}$ ) or $13.2 \mathrm{mmol}$ (for $3 \mathbf{k ,}, \mathbf{l}, \mathbf{n}$ ), $1.6 \mathrm{M}$ in hexanes) was added to a cooled solution of $2(6 \mathrm{mmol})$ in anhydrous THF $(30 \mathrm{~mL})$ at $-78{ }^{\circ} \mathrm{C}$ under nitrogen. The mixture was stirred one hour (for $\mathbf{3 m}$ ) or overnight (for $\mathbf{3 k}, \mathbf{l}, \mathbf{n}$ ) at room temperature and then cooled to $-78{ }^{\circ} \mathrm{C}$. The solution of 1-cyanobenzotriazole $(0.91 \mathrm{~g}, 6.3 \mathrm{mmol})$ was added dropwise. The mixture was allowed to warm to room temperature, stirred overnight and poured into a saturated solution of ammonium chloride $(60 \mathrm{~mL})$. The product was extracted with ethyl acetate $(30 \mathrm{~mL} \times 3)$, the organic extracts combined, washed with brine, and dried over $\mathrm{MgSO}_{4}$. After evaporation, the residue was purified by column chromatography $\left(\mathrm{CH}_{2} \mathrm{Cl}_{2}\right.$ or heptane/ethyl acetate: 10/1) to afford the desired nitriles $3 \mathbf{k}-\mathbf{n}$. 
2,2-Diphenylacetonitrile (3k). White microcrystals (60\%), mp 73-74 ${ }^{\circ} \mathrm{C}$ (lit. ${ }^{21} \mathrm{mp} 72.0-73.5$ $\left.{ }^{\circ} \mathrm{C}\right) ;{ }^{1} \mathrm{H}$ NMR $\delta 7.41-7.26(\mathrm{~m}, 10 \mathrm{H}), 5.15(\mathrm{~s}, 1 \mathrm{H}) ;{ }^{13} \mathrm{C}$ NMR $\delta 135.8,129.2,128.2,127.7,119.7$, 42.6. Anal. Calcd for $\mathrm{C}_{14} \mathrm{H}_{11} \mathrm{~N}: \mathrm{C}, 87.01 ; \mathrm{H}, 5.74 ; \mathrm{N}, 7.25$. Found: C, 86.83; H, 5.80; N, 7.22.

2-(2-Pyridinyl)propanenitrile (3l). ${ }^{22}$ Yellow oil (70\%); ${ }^{1} \mathrm{H}$ NMR $\delta 8.60$ (br d, $\left.J=4.8 \mathrm{~Hz}, 1 \mathrm{H}\right)$, 7.75 (td, $J=7.7,1.8 \mathrm{~Hz}, 1 \mathrm{H}), 7.46$ (d, $J=7.8 \mathrm{~Hz}, 1 \mathrm{H}), 7.27$ (dd, $J=7.6,5.0 \mathrm{~Hz}, 1 \mathrm{H}), 4.08$ (q, $J$ $=7.3 \mathrm{~Hz}, 1 \mathrm{H}), 1.72(\mathrm{~d}, J=7.3 \mathrm{~Hz}, 3 \mathrm{H}) ;{ }^{13} \mathrm{C}$ NMR $\delta 156.0,149.8,137.4,122.9,121.0,120.9$, 33.7, 19.6; Anal. Calcd for $\mathrm{C}_{8} \mathrm{H}_{8} \mathrm{~N}_{2}$ : C, 72.70; H, 6.10; N, 21.20. Found: C, 72.46; H, 6.27; N, 21.25 .

5-Ethyl-2-furonitrile (3m). Yellow oil (65\%), bp 59-61 ${ }^{\circ} \mathrm{C} / 1 \mathrm{mmHg}$ (lit. ${ }^{23}$ bp $59-60{ }^{\circ} \mathrm{C} / 1$ $\mathrm{mmHg}) ;{ }^{1} \mathrm{H}$ NMR $\delta 7.01(\mathrm{~d}, J=3.6 \mathrm{~Hz}, 1 \mathrm{H}), 6.13(\mathrm{~d}, J=3.6 \mathrm{~Hz}, 1 \mathrm{H}), 2.70(\mathrm{q}, J=7.5 \mathrm{~Hz}, 2 \mathrm{H})$, $1.27(\mathrm{t}, J=7.5 \mathrm{~Hz}, 3 \mathrm{H}) ;{ }^{13} \mathrm{C} \mathrm{NMR} \delta 163.6,126.1,124.3,123.1,106.2,21.6,11.6$.

1-Methyl-1H-pyrrole-2-carbonitrile (3n). Yellow oil (55\%), bp 60-62 ${ }^{\circ} \mathrm{C} / 20 \mathrm{mmHg}$, (lit. ${ }^{24} \mathrm{bp}$ $\left.{ }^{60-62}{ }^{\circ} \mathrm{C} / 20 \mathrm{mmHg}\right) ;{ }^{1} \mathrm{H}$ NMR $\delta 6.81-6.78(\mathrm{~m}, 2 \mathrm{H}), 6.16(\mathrm{dd}, J=4.0,1.4 \mathrm{~Hz}, 1 \mathrm{H}), 3.78(\mathrm{~s}, 3 \mathrm{H})$. ${ }^{13} \mathrm{C}$ NMR $\delta 160.6,127.4,119.9,113.7,109.4,35.2$.

\section{Acknowledgments}

The authors are indebted to Dr. Rachel M. Witek and Dr. Dennis Hall (University of Florida) for help and useful discussions during the preparation of this manuscript.

\section{References}

1. Hughes, T. V.; Cava, M. P. J. Org. Chem. 1999, 64, 313.

2. (a) Kahne, D.; Collum, D. B. Tetrahedron Lett. 1981, 22, 5011. (b) Van Leusen, A. M.; Jagt, J. C. Tetrahedron Lett. 1970, 12, 967.

3. Davis, W. A.; Cava, M., P. J. Org. Chem. 1983, 48, 2774.

4. Boltze, K.-H.; Dell, H.-D.; Jansen, H. Liebigs Ann. Chem. 1967, 709, 63.

5. Kim, J.-J.; Kweon, D.-H.; Cho,S.-D.; Kim,H.-K.; Jung, E.-Y.; Lee, S.-G.; Falck, J. R.; Yoon, Y.-J. Tetrahedron 2005, 61, 5889.

6. Wheland, R. C.; Martin, E. L. J. Org. Chem. 1975, 40, 3101.

7. Wu, Y.; Limburg, D. C.; Wilkinson, D. E.; Hamilton, G. S. Org. Lett. 2000, 2, 795.

8. Katritzky, A., R.; Brzezinski, J. Z.; Lam, J. N. Rev. Roum. Chim. 1991, 36, 573.

9. Hughes, T. V.; Hammond, S. D.; Cava, M. P. J. Org. Chem. 1998, 63, 401.

10. Meyers, M. J.; Sun, J.; Carlson, K. E.; Marriner, G. A.; Katzenellenbogen, B. S.; Katzenellenbogen, J. A. J. Med. Chem. 2001, 44, 4230.

11. Wildeman, J.; van Leusen, A. M. Synthesis 1979, 733.

12. Venier, C. G.; Squires, T. G.; Chen, Y.-Y.; Hussmann, G. P.; Shei, J. C.; Smith, B. F. J. Org. Chem. 1982, 47, 3773. 
13. Garden, S. J.; Guimarães, C. R. W.; Corréa, M. B.; de Oliveira, C. A. F.; Pinto, A. C.; de Alencastro, R. B. J. Org. Chem. 2003, 68, 8815.

14. You, J.; Verkade, J. G. J. Org. Chem. 2003, 68, 8003.

15. Leclerc, V.; Fourmaintraux, E.; Depreux, P.; Lesieur, D.; Morgan, P.; Howell, H. E.; Renard, P.; Caignard, D.-H.; Pfeiffer, B.; Delagrange, P.; Guardiola-Lemaître, B.; Andrieux, J. Bioorg. Med. Chem. 1998, 6, 1875.

16. Abd-El-Aziz, A. S.; de Denus, C. R. J. Chem. Soc., Perkin Trans. 1 1993, 293.

17. Fujii, M.; Nakamura, K.; Mekata, H.; Oka, S.; Ohno, A. Bull. Chem. Soc., Jpn. 1988, 61, 495.

18. Kuchař, M.; Brůnová, B.; Rejholec, V.; Jelínková, M.; Holubek, J.; Němeček, O. Collect. Czech. Chem. Commun. 1984, 49, 122.

19. Levine, R.; Hauser, C. R. J. Am. Chem. Soc. 1946, 68, 760.

20. Hagedorn, I.; Tönjes, H.; Pharmazie 1957, 12, 567.

21. Freeman, S. K.; Ringk, W. F.; Spoerri, P. E. J. Am. Chem. Soc. 1947, 69, 858.

22. Yoneda, R.; Harusawa, S.; Kurihara, T. Tetrahedron Lett. 1989, 30, 3681.

23. Pevzner, L. M. Russ. J. Gen. Chem. (Engl. Transl.) 2004, 74, 860.

24. Smaliy, R. V.; Chaikovskaya, A. A.; Pinchuk, A. M.; Tolmachev, A. A. Synthesis 2002, 2416. 